\title{
rassta: Raster-based Spatial Stratification Algorithms
}

\author{
by Bryan A. Fuentes ${ }^{1}$, Minerva J. Dorantes ${ }^{2}$, and John R. Tipton ${ }^{3}$
}

\begin{abstract}
Spatial stratification of landscapes allows for the development of efficient sampling surveys, the inclusion of domain knowledge in data-driven modeling frameworks, and the production of information relating the spatial variability of response phenomena to that of landscape processes. This work presents the rassta package as a collection of algorithms dedicated to the spatial stratification of landscapes, the calculation of landscape correspondence metrics across geographic space, and the application of these metrics for spatial sampling and modeling of environmental phenomena. The theoretical background of rassta is presented through references to several studies which have benefited from landscape stratification routines. The functionality of rassta is presented through code examples which are complemented with the geographic visualization of their outputs.
\end{abstract}

\section{Introduction}

The application of robust, quantitative approaches for the spatial modeling of environmental phenomena has increased in the past few decades mainly due to an increase in computational power, advances in statistical modeling, and the availability of geospatial layers of environmental information (Scull et al., 2003; Elith and Leathwick, 2009). Most of these approaches aim at building explicit quantitative relationships between environmental controls and response phenomena through statistical learning. Examples of these approaches include digital soil mapping (DSM) (McBratney et al., 2003), species distribution modeling (SDM) (Guisan and Zimmermann, 2000), land use/land cover classification (Ham et al., 2005), and forest fire modeling (Chuvieco et al., 2010). Despite the extensively documented success of these approaches, there are still some challenges that limit their application. For instance, poor statistical performance is often reported in studies where input data is too limited to accurately represent control-response relationships (Araújo and Guisan, 2006). Moreover, model parsimony and interpretation of results can be compromised when using 'black-box' algorithms (Arrouays et al., 2020). Similarly, including a priori knowledge about natural processes in purely statistical approaches can be challenging to achieve (Heuvelink and Webster, 2001).

Several studies have suggested embedding spatial stratification routines within approaches such as DSM, SDM, land use/cover mapping, forest fire modeling, and others to overcome the challenges limiting their application. In such studies, the spatial stratification of landscapes creates units with reduced spatial variability of environmental phenomena as compared to the overall variability across a landscape. The use of these units allows the researcher to (i) obtain balanced representations of control-response relationships (Guisan and Zimmermann, 2000; West et al., 2016); (ii) include expert knowledge of physical processes for improving modeling with limited data (Zhu, 1997); (iii) improve the performance of parameterization of mechanistic models (Park and Van De Giesen, 2004; Baldwin et al., 2017); and, (iv) facilitate the interpretation of environmental conditions and their influence on the spatiotemporal variability of processes of interest (Rodrigues et al., 2019).

In general, landscape stratification routines follow fundamental ecological concepts that explain the hierarchical and multi-scale nature of relationships between environmental phenomena across space (Allen and Starr, 1982). Therefore, landscape stratification methods have been applied in many studies that use geospatial information for environmental modeling, such as those previously cited. However, few packages exist in the $\mathrm{R}$ environment with functions strictly aimed at landscape stratification routines using geospatial data. Although one could implement custom stratification algorithms using multiple all-purpose geospatial analysis packages such as terra (Hijmans, 2021) and sf (Pebesma, 2018), the ease of use, reproducibility, and replicability of analysis is often enhanced when algorithms are implemented as part of a dedicated package. The motif package (Nowosad, 2021) is the only example the authors could find of a package that is fully dedicated to landscape stratification in $\mathrm{R}$ using geospatial data. Although the methods offered by motif are effective for large-scale studies (Jasiewicz et al., 2015; Netzel and Stepinski, 2015; Nowosad, 2021), their application is currently limited to rasters of categorical data. Thus, motif is not practical for the modeling of spatially continuous environmental phenomena, which is often a goal of landscape stratification routines.

This work presents the rassta package as a collection of algorithms for the spatial stratification of landscapes, sampling, and modeling of environmental phenomena. The rassta package is not intended as a drop-in replacement for statistically-robust environmental modeling approaches. Rather, it is intended to serve as a generalized framework to derive geospatial information that can be used to improve inference with these statistical approaches.

\footnotetext{
${ }^{1}$ University of Arkansas - Department of Crop, Soil, and Environmental Sciences - bafuente@uark.edu

${ }^{2}$ University of Arkansas - Department of Crop, Soil, and Environmental Sciences - mjdorant@uark.edu

${ }^{3}$ University of Arkansas - Department of Mathematical Sciences - jrtipton@uark.edu
} 


\section{Conceptual overview and functionality}

The algorithms in the rassta package assist in the analysis of environmental information related to the spatial variability of natural phenomena across landscapes. These functions focus on integrating standard geospatial techniques and quantitative analysis in a generalized framework for landscape stratification, sampling, and modeling. All of the functions in the rassta package take geospatial data in raster format as input. In the context of geographic information systems (GIS), the raster format can be considered a graphical representation of a matrix that is organized in rows and columns, and which may be stacked in multiple layers (e.g., multi-band satellite imagery). Each cell (pixel) in the raster contains a value representing a spatially-varying phenomenon, such as elevation or precipitation. A few functions in rassta also produce geospatial data in vector format. Vector data represents geometric entities in the form of points, lines, and polygons. The rassta package uses the highly efficient terra package as the backbone for handling raster and vector data. Most of the geospatial data manipulation with terra is performed in $\mathrm{C}++$ and is based on two main $\mathrm{R}$ data types (classes): SpatRaster and SpatVector.

Most of the functions implemented in rassta are interrelated in the sense that the outputs from some functions can be used as the inputs for others. This functional interrelation allows for a generalized framework to conduct spatial stratification, sampling, and modeling in a single package following a project-oriented approach. In general, the functions of rassta can be grouped into five categories: (1) landscape stratification, (2) landscape correspondence metrics, (3) stratified sampling, (4) spatial modeling, and (5) miscellaneous (Figure 1). Each category and its corresponding functions (except for miscellaneous) are theoretically founded on several studies focused on understanding spatiallyvarying natural phenomena across landscapes. In the next sections, the rationale behind each category and its functions is described. This description is complemented with references to corresponding scientific literature and includes code examples showing the application of each function with extensive use of plotting functions (for visualization purposes only). Most of the plotting functions are derived from the terra package using the SpatRaster and SpatVector classes. [Note: To reduce the extension of code examples, all the map and graph plotting functions were consolidated in the function figure()].

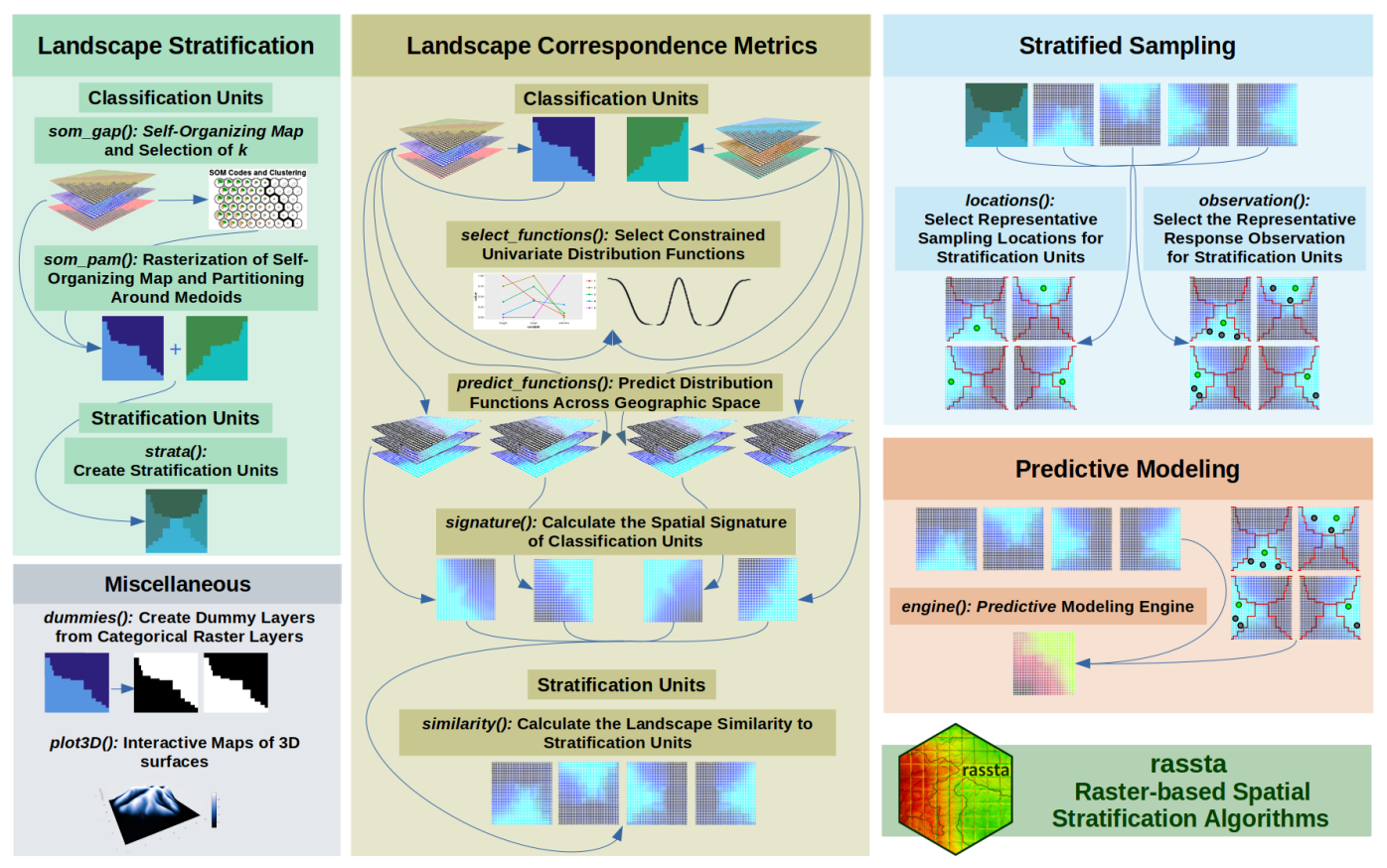

Figure 1: Functions of the rassta package. Connectors relate the inputs and outputs of the functions.

\section{Landscape stratification}

Several studies have suggested the need to account for the hierarchical and multi-scale nature of landscape processes. Allen and Starr (1982) suggested that landscape processes can be explained through hierarchical multivariate structures given their multiple spatial and temporal scales. Based on Dokuchaeiv's theory of soil formation (Glinka, 1927) and the soil-landscape paradigm (Hudson, 1992), McSweeney et al. (1994) proposed a nested model of soil-landscape processes at the physiographic, geomorphometric, and within-soil domains. Flügel (1995) suggested that the regionalization of 
hydrology-related processes should consider the multi-scale landscape heterogeneity in terms of soil, topography, geology, climate, and vegetation. These ideas have led these and other authors to formulate frameworks for the creation of spatial entities that stratify the landscape. The general purpose of these entities has been to define spatially-explicit domains that represent distinctive landscape processes and/or interactions (McSweeney et al., 1994). Accordingly, spatial stratification using rassta focuses on the creation of such domains (hereafter referred to as units).

The landscape stratification process with rassta follows a hierarchical approach similar to Austin and Heyligers (1989), who individually classified gradients of precipitation and elevation into intervals that were intersected with geologic classes for sampling purposes. Similarly, in rassta, a set of first-level units is created separately for each landscape factor under analysis. Then, multiple sets of first-level units are integrated into a single set of second-level units. The first-level units, called classification units, can be created outside of rassta via multi-criteria analysis, statistical learning, or other methods. Moreover, the classification units can be formally defined through classification schemes, such as those based on taxonomic keys. The second-level units, called stratification units, result from the spatial intersection of multiple sets of classification units. Note that both classification and stratification units represent a spatial stratification for a given landscape.

A set of $n$ classification/stratification units represents $n$ distinct landscape configurations related to a single landscape factor (classification unit), or to multiple landscape factors (stratification unit). Note that the term landscape configuration is used as a generic term for a particular pattern in the spatial variability of one or multiple variables belonging to one or multiple landscape factors. Figure 2 shows an example of a simple landscape stratification process based on two landscape factors, each with three raster layers representing continuous variables.

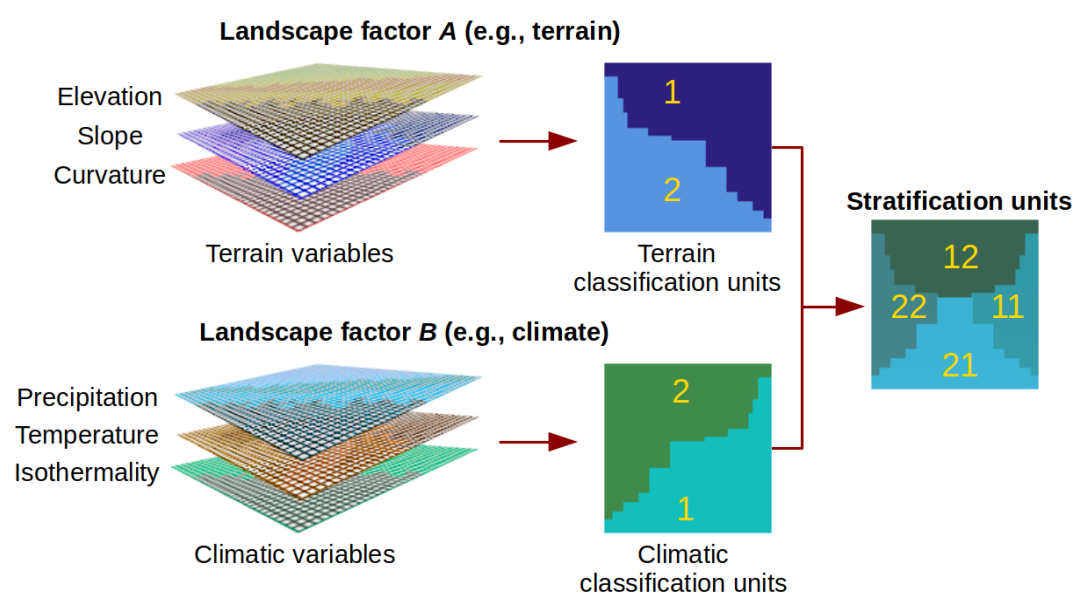

Figure 2: Schematic of a landscape stratification process.

There are three important aspects of the stratification approach used within rassta that must be considered. (1) One can simply create stratification units by incorporating variables from multiple landscape factors in a single classification process. However, the interpretation of results is often compromised when using a large number of variables in "all-in-the-bag" statistically driven classification schemes. (2) Multiple sets of classification units can belong to a single landscape factor, and each set can be created from variables at a distinct spatial scale. Presumably, this can account for the multi-scale nature of landscape factors in the stratification process. (3) A landscape factor can be represented by a single categorical variable, as in the case of geologic units or soil parent material. In this case, the landscape factor/variable is already in the form of classification units. Figure 3 shows a landscape stratification scenario like that addressed in (2) and (3). 


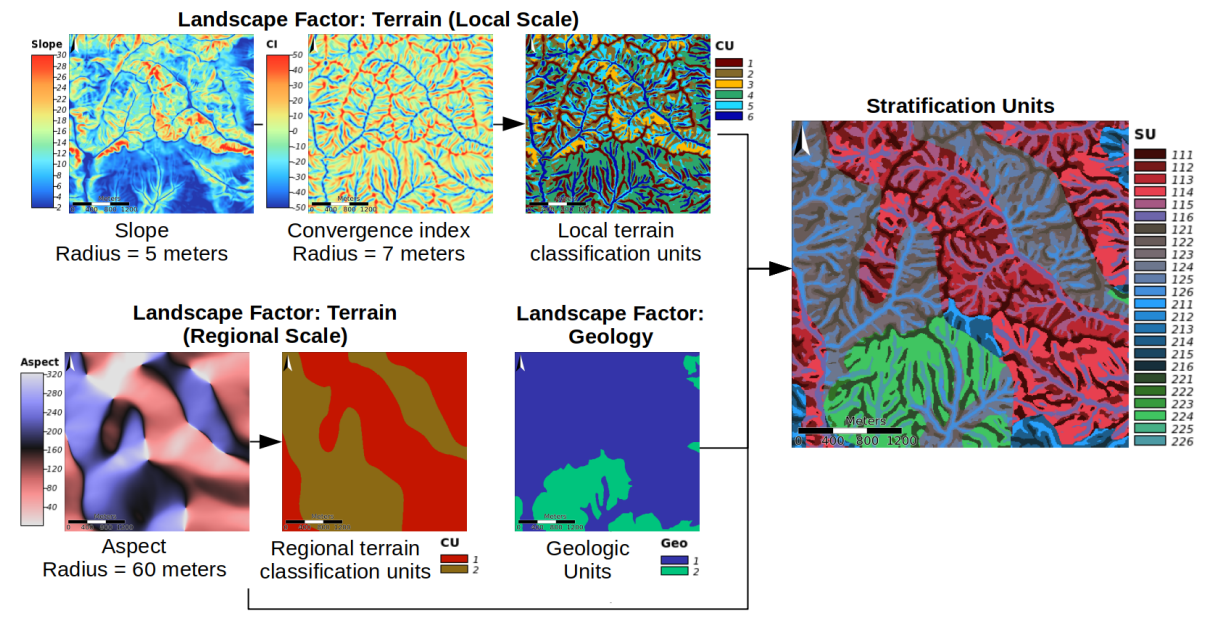

Figure 3: Landscape stratification based on one multi-scale and one categorical landscape factor.

Currently, rassta allows the creation of classification units via unsupervised learning thanks to the functions som_gap () and som_pam(). The function som_gap() performs dimension reduction based on the self-organizing map (SOM) proposed by Kohonen (1990) and implemented in R through the kohonen package (Wehrens and Kruisselbrink, 2018). Moreover, som_gap() performs cluster analysis on the SOM codes based on the partitioning around medoids (PAM) (Kaufman and Rousseeuw, 1990), with estimation of the optimum number of clusters $(k)$ through the gap statistic (Tibshirani et al., 2001). It is important to mention that the output SOM object can be used as input for any other clustering algorithm (e.g., hierarchical, spectral, etc.) or statistical analysis outside of rassta.

The code below shows how som_gap () reduces the feature space and selects $k$ clusters from four terrain variables.

\# Load the rassta and terra packages

library (rassta)

library(terra)

\# Get the data required to run the examples from rassta's installation folder wasoil <- system.file("exdat/wasoil.zip", package = "rassta")

file. $\operatorname{copy}($ from $=$ wasoil, to $=\operatorname{getwd}())$ \# Copy to current working directory unzip("wasoil.zip") \# Extract files

\section{\# Set seed}

set.seed(963)

\# Multi-layer SpatRaster with 4 terrain variables

terr.var <- rast(c("height.tif", "midslope.tif", "slope.tif", "wetness.tif"))

\# Scale variables to mean $=0$ and standard deviation $=1$

terr.varscale <- scale(terr.var)

\# Dimensionality reduction and estimation of optimum $\mathrm{k}$ (max $\mathrm{k}$ to evaluate: 12)

terr.som <- som_gap(terr.varscale, $x$ dim $=10$, ydim $=10, \mathrm{~K} . \max =12$ )

figure(4, $d=$ list(terr.var, terr.som)) \# Plot results

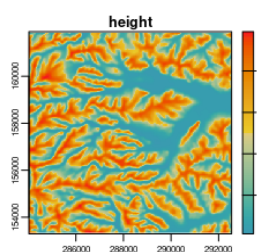

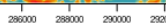

som - height

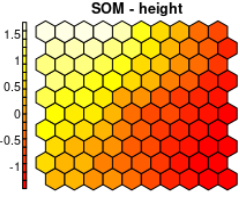

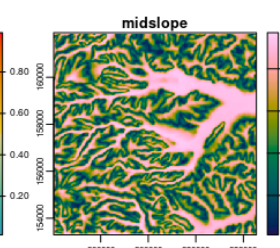

250mo 2500000

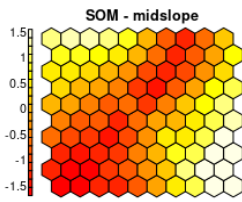

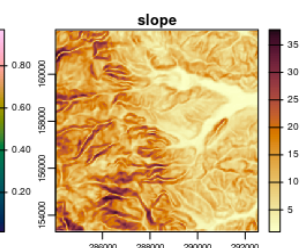

som - slope

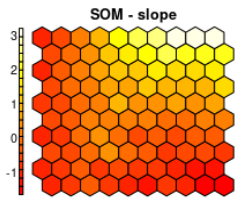

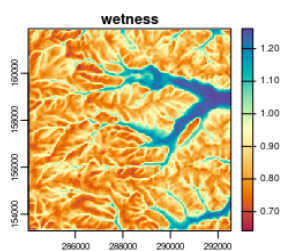

som - wetness

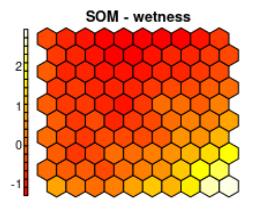

som - Codes

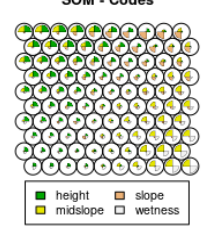

Gap Statistic

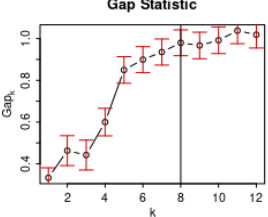

Figure 4: Terrain variables, SOM codes and components (variables), and Gap statistic for PAM. 
The function som_pam() creates raster versions from the outputs of som_gap(). The code below shows how som_pam() creates raster versions of the SOM grid and PAM clustering computed in the previous example.
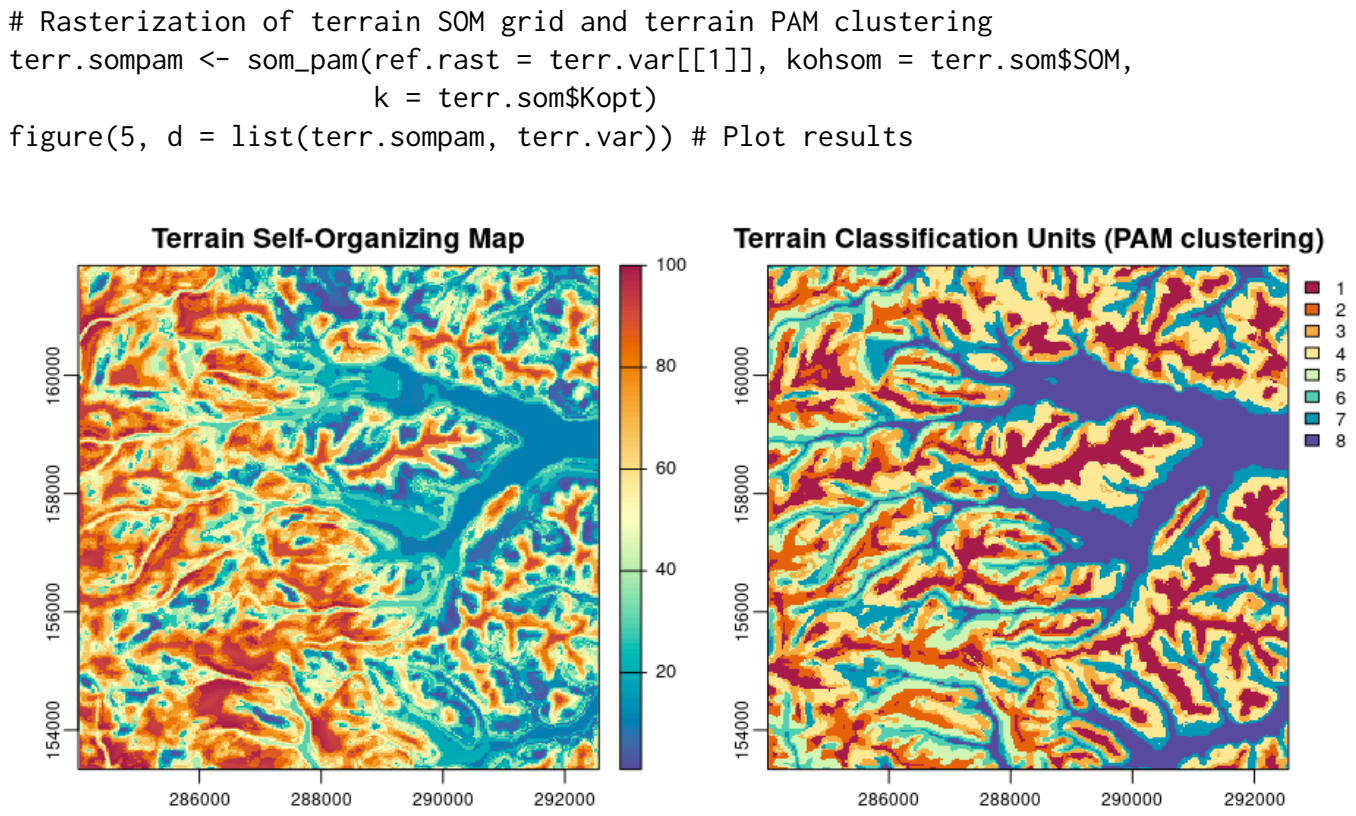

Figure 5: Rasterized terrain SOM grid and terrain PAM clustering.

Note that the approach for creating classification units should not be limited to that offered by som_gap () and som_pam(). There are many other approaches outside of rassta that can be followed, such as supervised classification based on statistical learning, or GIS-based multi-criteria analysis. The best approach may depend on the research question(s) being addressed. Therefore, the selection of the proper approach and the optional use of other R packages and/or GIS software is left to the user.

The function strata() allows the spatial intersection of multiple sets of classification units into a single set of stratification units. This function also assigns a unique numeric code to each stratification unit. The numeric code makes it possible to trace back each classification unit composing a given stratification unit. The code below shows the construction of stratification units with strata() using classification units from three landscape factors (climate, soil parent material, and terrain).

\# Multi-layer SpatRaster with 3 sets of classification units

all.cu <- rast(c("climate.tif", "material.tif", "terrain.tif"))

\# Stratification units

su <- strata(cu. rast $=$ all.cu)

figure $(6, \mathrm{~d}=\operatorname{list}(\mathrm{su}, \mathrm{all} . \mathrm{cu}))$ \# Plot results
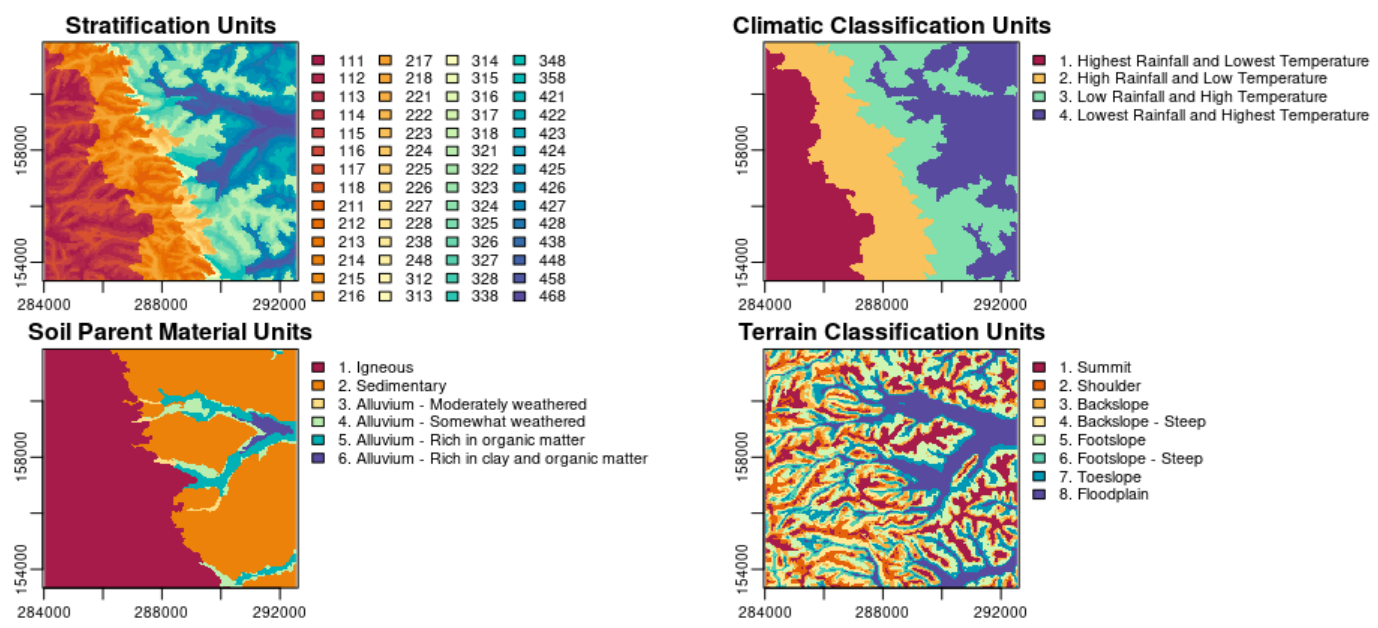

Figure 6: Stratification units from climate, soil parent material, and terrain classification units. 


\section{Metrics of landscape correspondence}

There are two metrics of landscape correspondence that can be calculated with rassta: (1) the spatial signature of classification units, and (2) the landscape similarity to stratification units. These metrics quantify the relative correspondence between any location across geographic space and landscape configurations represented by classification and stratification units. Several studies have applied similar concepts related to continuous correspondence between landscape configurations for the modeling of spatially-varying phenomena. Early examples include studies using multivariate distance metrics in the feature space for SDM (Carpenter et al., 1993) and studies applying the fuzzy set theory (Zadeh, 1965) for multi-criteria evaluation (Burrough, 1989; Sui, 1992), DSM (Zhu, 1997) and landform classification (MacMillan et al., 2000).

\section{Spatial signature of classification units}

The spatial patterns of the degree of correspondence between any landscape configuration and the configuration represented by a given classification unit are defined as the spatial signature. The spatial signature is represented by a raster layer of continuous values that results from the cell-wise aggregation of empirical distribution functions mapped over geographic space. Each distribution function corresponds to one variable and relates the classification unit to "typical" values of the variable within the classification unit. The concept of spatial signature is based on the work of Pike and Rozema (1975) and Pike (1988). These authors used the term geometric signature to describe a set of sample statistics (e.g., mean, standard deviation) of terrain variables (e.g., slope, curvature) used to distinguish "geomorphically disparate landscapes" (Pike, 1988).

The spatial signature in rassta replaces the geometric signature's measurements of central tendency and dispersion statistics with statistical distribution functions generated across geographic space. The statistical distribution functions used in rassta are: (1) the probability density function (PDF) based on the kernel density estimation, (2) the empirical cumulative distribution function (ECDF), and (3) an inverted version of the ECDF (iECDF). Note that the spatial signature concept is somewhat similar to the virtual ecological niche (Hirzel et al., 2001) and the multivariate environmental similarity surface (Elith et al., 2010), which are implemented in R through the packages virtualspecies (Leroy et al., 2016) and dismo (Hijmans et al., 2020), respectively. Figure 7 shows an example of the process to calculate the spatial signature of one classification unit.

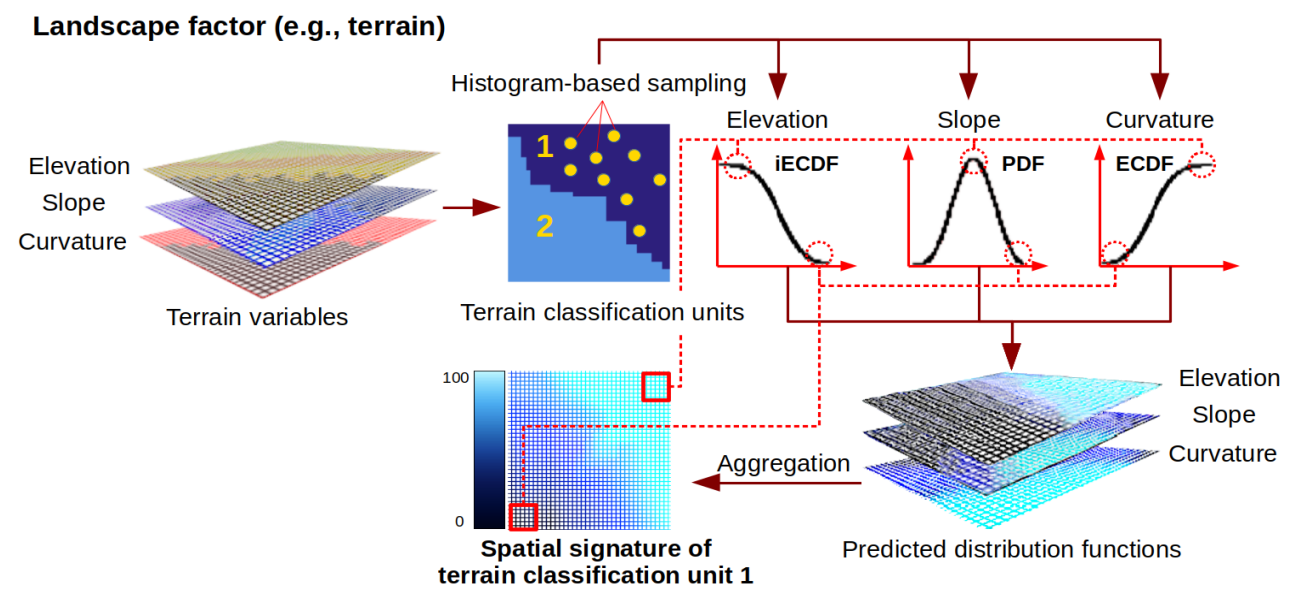

Figure 7: Schematic of the calculation process for spatial signatures. The red squares in the raster layer of terrain classification units represent raster cells.

An important assumption is made when using the PDF, ECDF, and iECDF to characterize the typical values of a given variable within a given classification unit. The position of a value within the distribution function is an indicator of how typical the value is in terms of the variable's distribution within the classification unit. For instance, values closer to, or at the peak of the PDF are assumed to be the most typical values of the variable within the classification unit. Contrarily, values at the tails of the PDF are the less typical. Although one could simply use the PDF as a generalized function to denote typical values, this function assigns the same weight to values at the tails of the distribution regardless of the tail's location (left or right). In some cases, a priori knowledge can dictate that typical values of a variable within a given classification unit are those approaching $+\infty$, or those approaching $-\infty$. The use of the ECDF and the iECDF is intended for those cases. More specifically, if a classification unit 
is known to be associated with a variable's extreme values toward $+\infty$, then the ECDF can be used to represent this association. Conversely, if the classification unit is associated with those variable's extreme values toward $-\infty$, then the iECDF can be used.

The function select_functions() allows the user to select the statistical distribution function used to represent the typical values for a given variable within a specific classification unit. Both automatic and interactive selection modes are supported, with the latter based on a shiny app (Chang et al., 2021). The automatic selection of distribution functions is based on within-class statistics, also referred to as zonal statistics in the GIS literature, and it follows the criteria described next:

- $\mathrm{PDF}=$ when the mean (or median) of the variable's values within the classification unit is neither the maximum nor the minimum of all the mean (or median) values across all the units.

- $\mathrm{ECDF}=$ when the mean (or median) of the variable's values within the classification unit is the maximum of all the mean (or median) values across all the units.

- $\mathrm{iECDF}=$ when the mean (or median) of the variable's values within the classification unit is the minimum of all the mean (or median) values across all the units.

The code below shows the automatic selection of statistical distribution functions for four climatic classification units and two variables with select_functions().
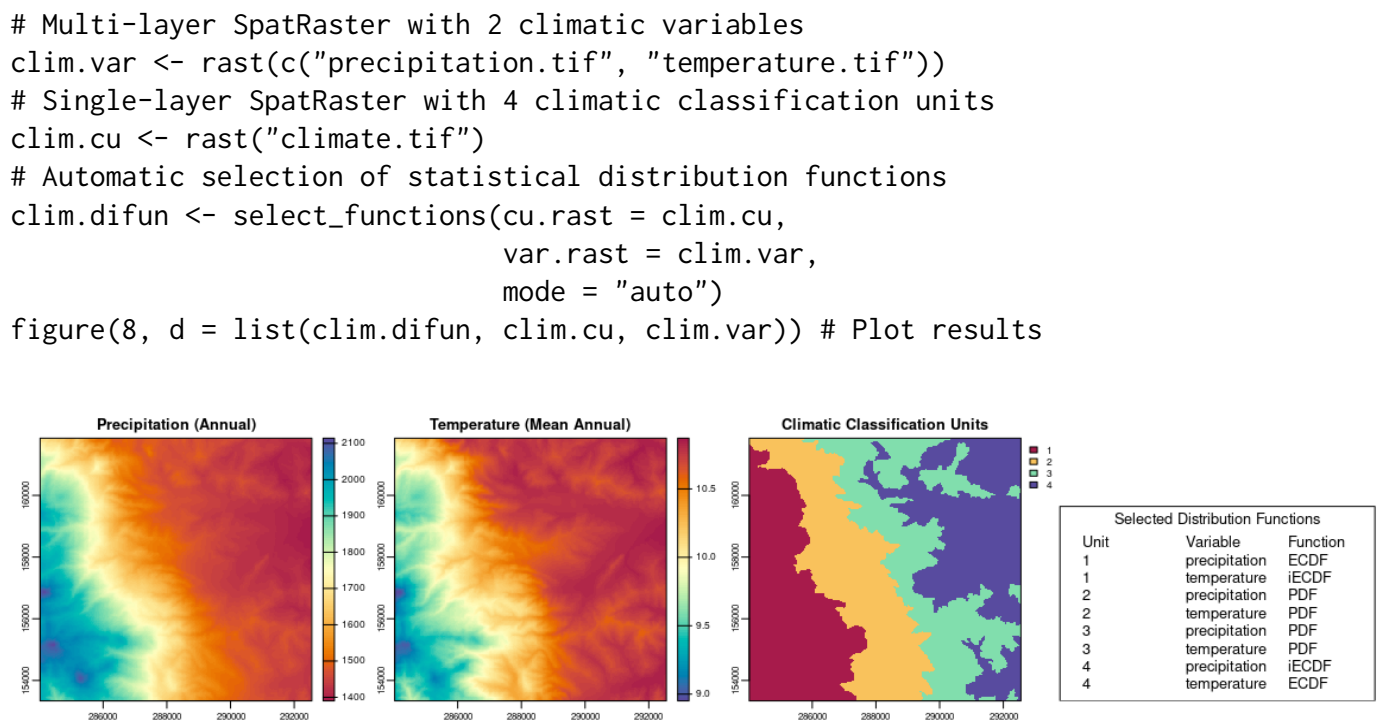

Figure 8: Climatic variables, classification units, and selected distribution functions per variable at each classification unit.

The selected distribution functions can be used to generate predictions of distribution function values over geographic space with the function predict_functions() as shown in the code below. The predictions are generated by fitting a locally estimated scatterplot smoothing (LOESS) regression with the within-unit distribution function's values (y) and the within-unit variable's values (x). The fitted LOESS and the raster layer of the variable are then used to predict new distribution function values across geographic space.

\# Multi-layer SpatRaster of climatic variables and classification units

clim.all <- c(clim.var, clim.cu)

\# Ouput table from select_functions()

df $<-$ clim.difun\$distfun

\# Predicted distribution functions for climatic variables

clim.pdif <- predict_functions(cuvar.rast = clim.all,

$\mathrm{cu}$. ind $=3$,

$\mathrm{cu}=\mathrm{df} \$$ Class. Unit,

vars $=\mathrm{df} \$$ Variable,

dif $=$ df $\$$ Dist.Func)

figure(9, d = list(clim.pdif, clim.cu)) \# Plot results 

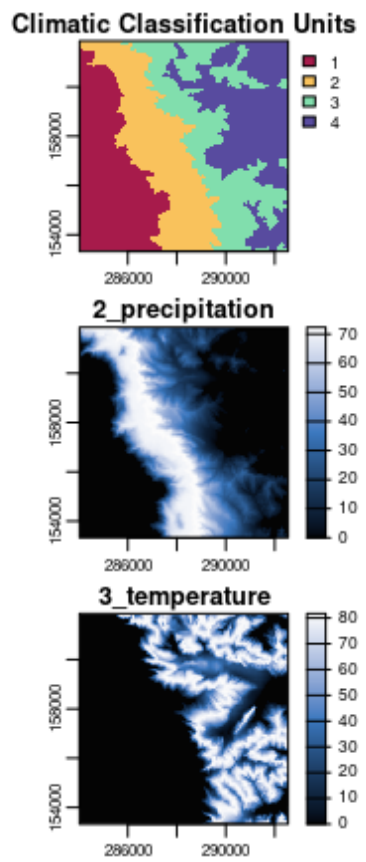

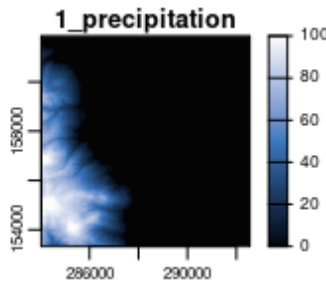

2 temperature
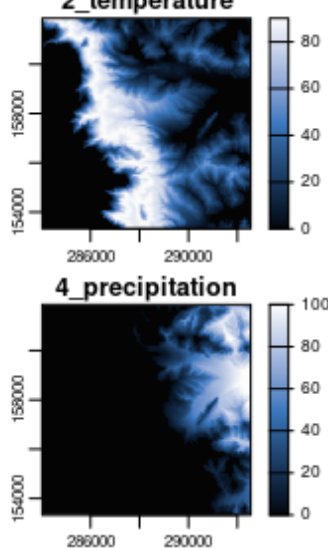

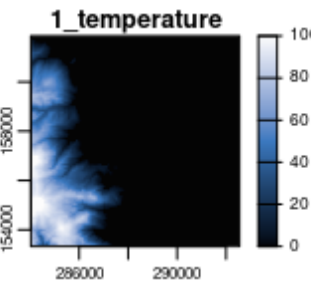

3 precipitation
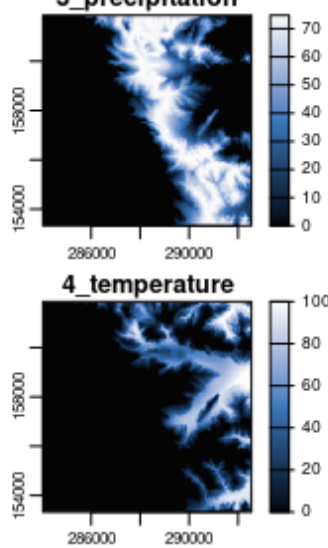

Figure 9: Climatic classification units and their corresponding predicted distribution functions for each variable.

The function signature() calculates the spatial signature of a given classification unit by aggregating all of the predicted distribution functions associated with the unit. The code below shows the calculation of spatial signatures with signature(). Note that the arguments inprex and outname allow the user to identify the raster layers representing the predicted distribution functions associated with each classification unit in a set, and to assign a unique name to each resulting raster layer of spatial signature, respectively.
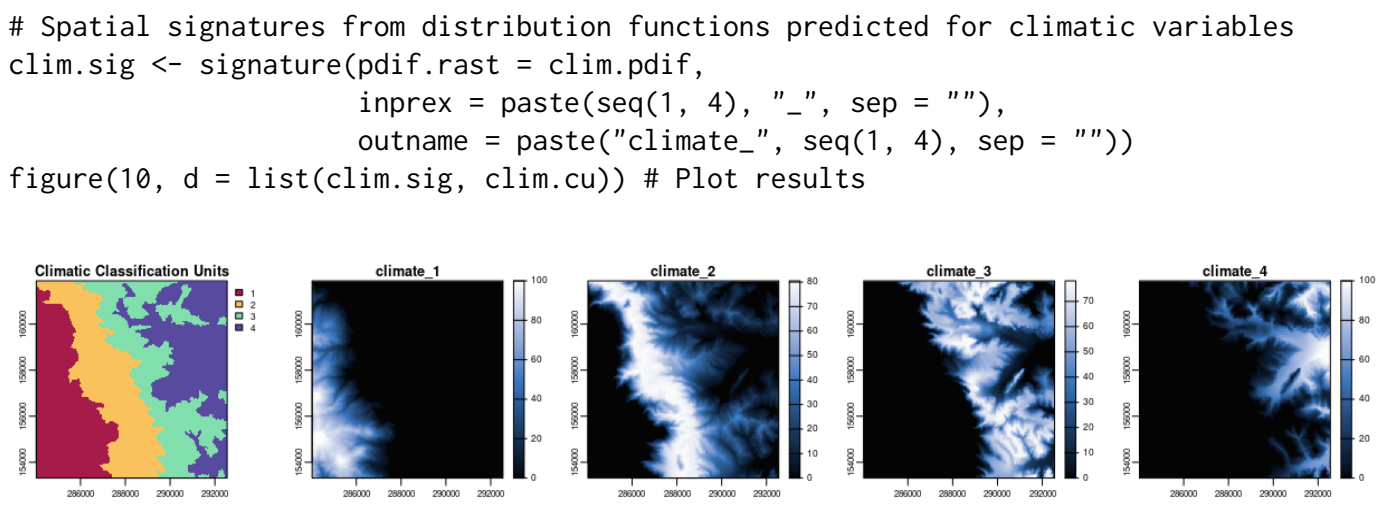

Figure 10: Climatic classification units and their corresponding spatial signatures.

\section{Landscape similarity to stratification units}

The spatial patterns of the degree of correspondence between any landscape configuration and the landscape configuration represented by a given stratification unit are defined as the landscape similarity. The landscape similarity is represented by a raster layer of continuous values, which results from the cell-wise aggregation of the spatial signatures of multiple classification units. This aggregation is possible because any given stratification unit is the result of the spatial intersection of multiple classification units, commonly one per landscape factor or factor scale (see Figures 2 and 3). Moreover, each classification unit has one spatial signature associated with it. Therefore, any given stratification unit will be associated with multiple spatial signatures, which can be cell-wise aggregated to calculate the landscape similarity. Figure 11 shows an example of the calculation process for the landscape similarity to stratification units. 


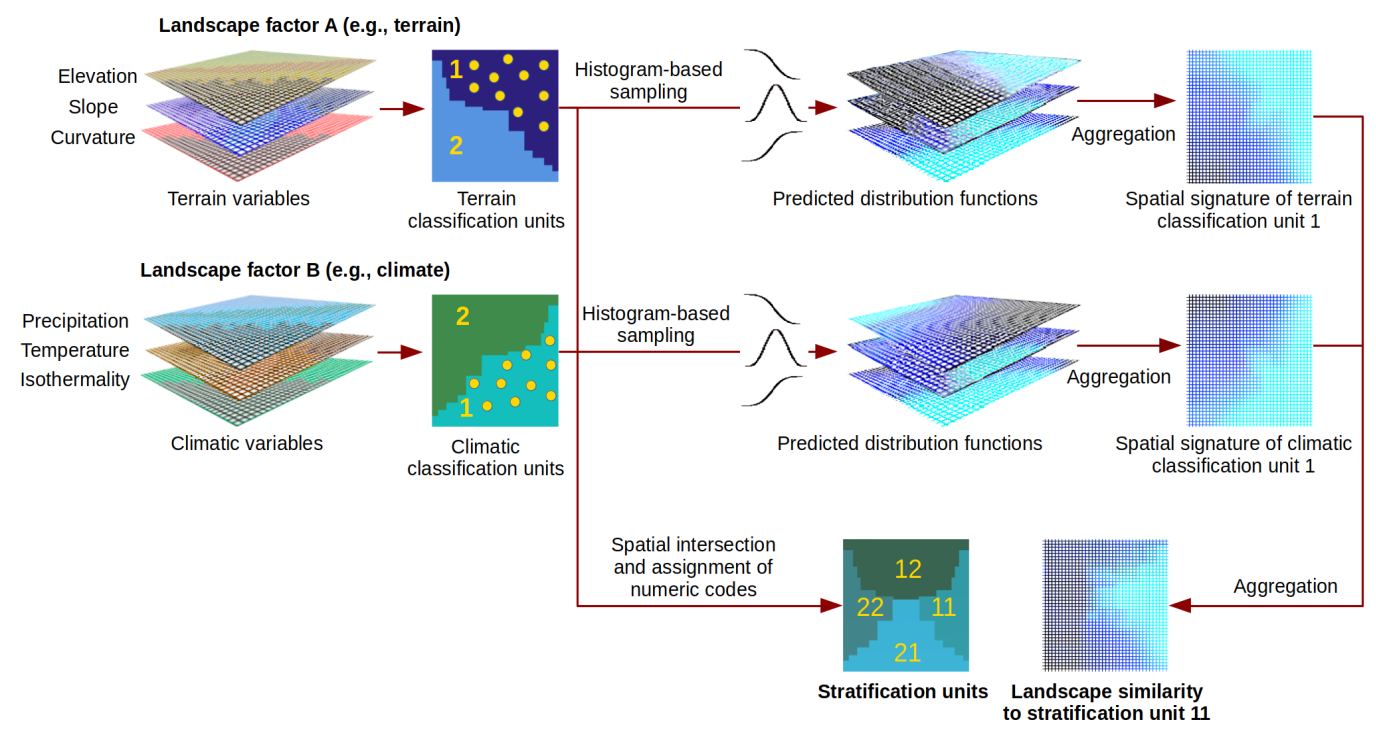

Figure 11: Schematic of the calculation process for landscape similarities.

The function similarity() calculates the landscape similarity for all of the stratification units in a given set (represented by a single-layer SpatRaster object) as shown in the following example.The argument su.code indicates the name of the landscape factors/factor scales used to create the stratification units, and the digit position (start, end) of the classification units' ID in the stratification unit's numeric code.

\# Multi-layer SpatRaster with spatial signatures of classification units clim.sig <- rast(list.files(pattern = "climate_")) \# For climatic units mat.sig <- rast(list.files (pattern = "material_")) \# For soil parent material units terr.sig <- rast(list.files(pattern = "terrain_")) \# For terrain units

\# Single-layer SpatRaster of stratification units

su <- rast("su.tif")

\# Landscape similarity to stratification units

su.ls $<-$ similarity (su.rast $=$ su, sig.rast $=c(c l i m . s i g$, mat.sig, terr.sig $)$, su. code $=$ list $($ climate $=c(1,1)$, material $=c(2,2)$, terrain $=c(3,3))$ )

figure(12, d = list(su.ls, su, clim.sig, mat.sig, terr.sig)) \# Plot results
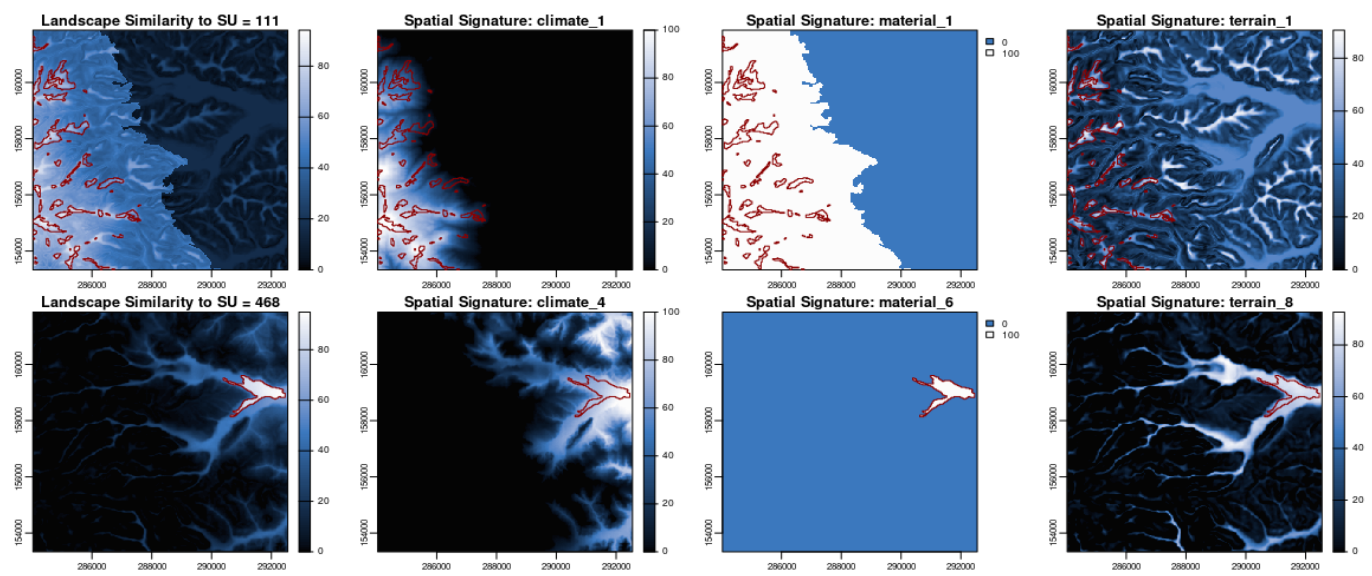

Figure 12: Landscape similarity to stratification unit (SU) 111 and 468, and spatial signatures from their corresponding classification units. Red polygons indicate the boundaries of SU 111 and 468.

\section{Stratified non-probability sampling}

Stratified sampling is an efficient technique for achieving an adequate representation of environmental variability, reducing cost of field work, and improving modeling with limited observations (Austin 
and Heyligers, 1989; Wessels et al., 1998; Guisan and Zimmermann, 2000; Zhu et al., 2008; West et al., 2016). Accordingly, sampling with rassta to select observations/sampling locations is performed in a stratified fashion using stratification units. Additionally, the raster layers of landscape similarity to stratification units can be included in the sampling process. Including the raster layers of landscape similarity results in a non-probability sample. For each stratification unit, the sampling process selects the observation(s)/sampling location(s) at the $X Y$ position where the highest landscape similarity value occurs, resulting in a stratified, non-probability sample that is biased towards maximizing the representativeness of landscape configurations. This idea of biased, stratified sampling is based on the work of Gillison (1983); Gillison and Brewer (1985), Austin and Heyligers (1989), and Zhu et al. (2008). These authors have suggested that bias related to landscape configurations is relevant for the maximization of environmental representativeness, detection of maximum diversity, and representation of non-stochastic control-response relationships.

The function observation() performs the automatic selection of the representative response observation for each stratification unit in a given set. Given a stratification unit, the unit's representative response observation is that whose value best reflects the influence that the unit's landscape configuration exerts on the response. This function requires a set of observations/samples already collected for a set of stratification units. Currently, observation() selects observations based on the following methods: (1) mls: select the observation at the location with the maximum landscape similarity value; (2) $m r v$ : select the observation whose response value is the median of all the values; and (3) random: select an observation at random. Note that the latter represents a case of stratified random sampling.

The code below shows the selection of representative soil organic carbon (SOC) observations based on the maximum landscape similarity method. Note that the arguments su.rast and ls.rast require the stratification units and landscape similarity layers previously created with strata() and similarity(), respectively.
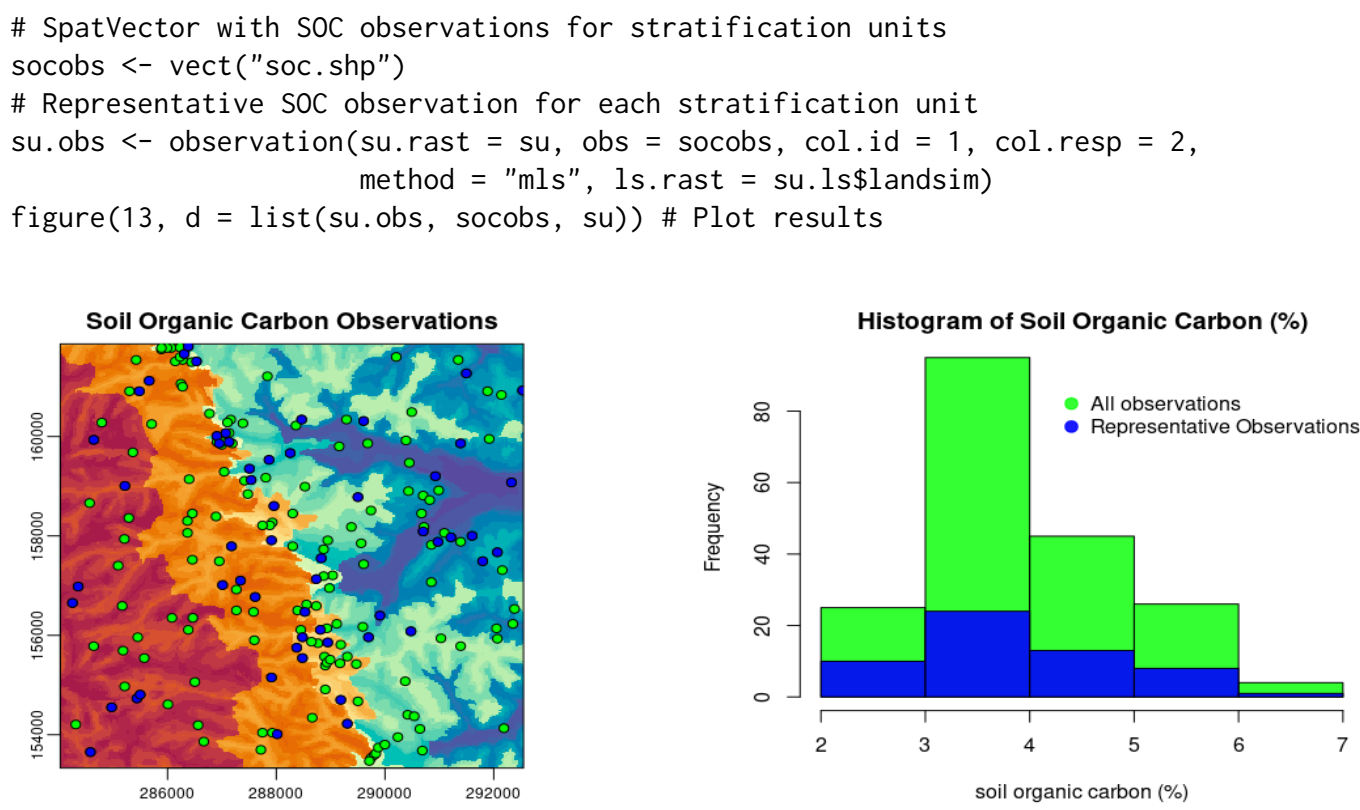

Figure 13: All observations and representative SOC observations for stratification units.

The function locations() performs the automatic selection of the representative sampling location(s) for each stratification unit in a given set, where the representative sampling location is the $X Y$ position where the highest landscape similarity value occurs. Currently, locations() implements two selection methods: (1) buffer: select $X Y$ locations within areas with landscape similarity values above a certain threshold; and (2) absolute: select $X Y$ locations with the highest landscape similarity values. The code below shows the use of locations() based on the buffer method.

\# Representative sampling location and its buffer area for each stratification unit su. samp <- locations (1s.rast $=$ su.ls $\$$ landsim, su.rast $=$ su, method $=$ "buffer")

figure(14, d = list(su.samp, su)) \# Plot results 

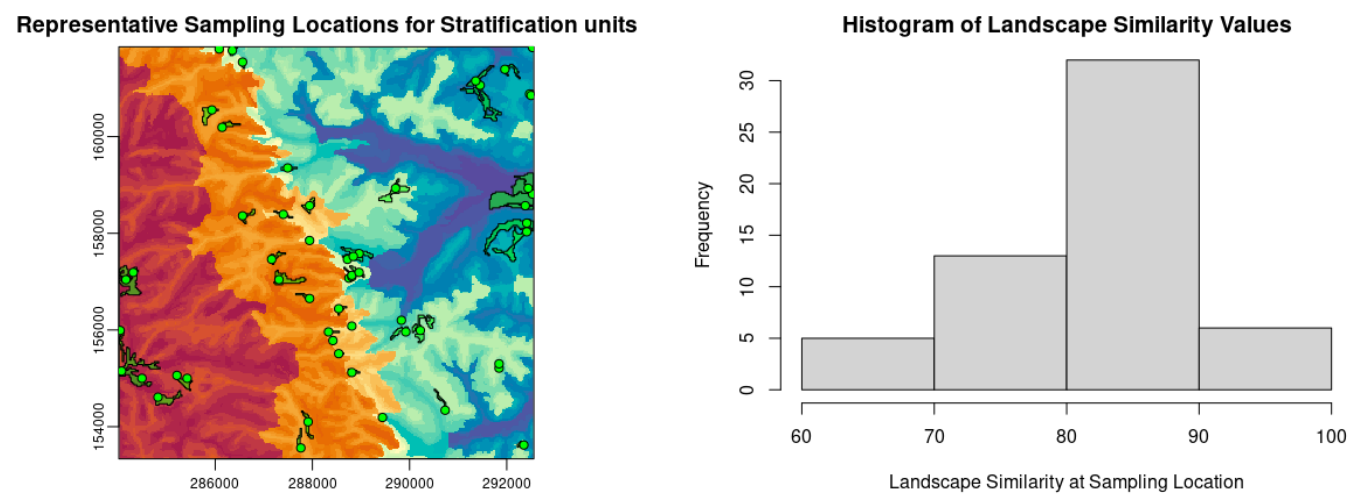

Figure 14: Representative sampling locations and their buffer areas for stratification units.

\section{Predictive modeling}

Predictive modeling with rassta is based on the assumption that each stratification unit represents a distinct landscape configuration and that this configuration influences a natural phenomenon in a distinctive manner. The influence that a stratification unit's landscape configuration has on response phenomena at a specific location is proportional to the unit's landscape similarity value at that location. Therefore, given a stratification unit $x$, the corresponding raster layer of landscape similarity $l s$, the location $X Y$, and the response $y$, the greater the value of $l s$ at $X Y$, the more similar $y$ at $X Y$ will be to the typical $y$ for $x$. The typical (i.e., representative) value of a response phenomenon for a given stratification unit can be defined in several ways. For instance, if a response phenomenon was sampled/measured multiple times within a given stratification unit, the typical response value could be that from the sample/measurement at the location with the highest landscape similarity value (see observation()). Several studies have used landscape similarity metrics within predictive modeling frameworks for natural phenomena. Early examples include those related to SDM based on environmental similarity (Carpenter et al., 1993; Knick and Dyer, 1997; Watrous et al., 2006), and those related to DSM based on fuzzy logic (Zhu and Band, 1994; Zhu, 1997; Schmidt et al., 2005; Zhu et al., 2010). Figure 15 shows an example of the spatial modeling process for continuous responses with rassta.

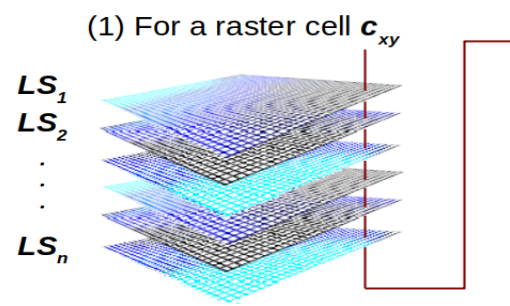

Raster layers of landscape similarity to stratification unit $L S_{1,2, \ldots, n}$

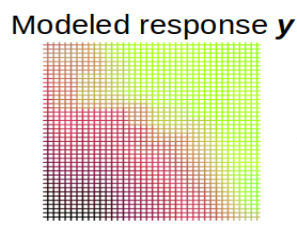

(2) Find the $\boldsymbol{n}$ highest landscape similarity values $L S$ $\operatorname{sort}\left(L S_{1} c_{x y}, L S_{2} c_{x y}, \ldots, L S_{n} c_{x y}\right)$

(3) Identify the $n$ stratification units $S U w_{1,2, \ldots, n}$ with the highest $\boldsymbol{L} \boldsymbol{S}$ and their corresponding representative response values $\boldsymbol{r}$

\begin{tabular}{ccc}
\hline SUW $_{1}$ & $Y_{1}$ & $L S_{1} c_{x y}$ \\
$\operatorname{SUW}_{2}$ & $Y_{2}$ & $L S_{2} c_{x y}$ \\
$\vdots$ & $\vdots$ & $\vdots$ \\
$\operatorname{SUW}_{n}$ & $Y_{n}$ & $L S_{n} c_{x y}$ \\
\hline & &
\end{tabular}

(4) Calculate the response value $y$

$$
y c_{x y}=\frac{\sum_{i=1}^{n} Y_{i} * L S_{i} c_{x y}}{\sum_{i=1}^{n} L S_{i} c_{x y}}
$$

Figure 15: Schematic of the spatial modeling process with rassta for continuous responses.

Predictive modeling of response phenomena with rassta is performed using the function engine(). For continuous responses, engine() performs a weighted average involving representative response values and landscape similarity values. For a location $X Y$, the modeled response value is equal to the weighted average of the representative values for those stratification units with the highest landscape similarity values at $X Y$. The stratification units with the highest landscape similarity values at $X Y$ can be considered as the nearest neighbors (in feature space) of the landscape configuration at 
$X Y$. These neighbors are called "winning" stratification units, and the weight of their corresponding representative value is proportional to the winning unit's landscape similarity value at $X Y$. For categorical responses, the modal response value of the winning stratification units replaces the weighted average.

The code below demonstrates the use of engine() for the predictive modeling of soil organic carbon. Note that the representative response values (argument su.repobs) are those previously selected with observation(), and that the layers of landscape similarity (argument ls.rast) are those previously created with similarity().

\# Table with the numeric code of stratification units and representative SOC values su.soc <- su.obs\$su_repobs[, c("SU", "soc")]

\# engine() requires a (tiled) SpatVector with the boundaries of the area of interest aoi <- vect("aoi.shp")

\# engine() writes results directly on disk

if (dir.exists ("soc") == FALSE) \{dir.create("soc")\} \# Create directory

\# Spatial modeling of SOC across the landscape based on 3 winning stratification units soc $<-$ engine(ls.rast $=$ su.ls $\$$ landsim, n.win $=3$, su.repobs $=$ su. soc , tiles = aoi, outdir = "soc", overwrite = TRUE)

figure(16, d = list(soc, "soc_valid.shp")) \# Plot results
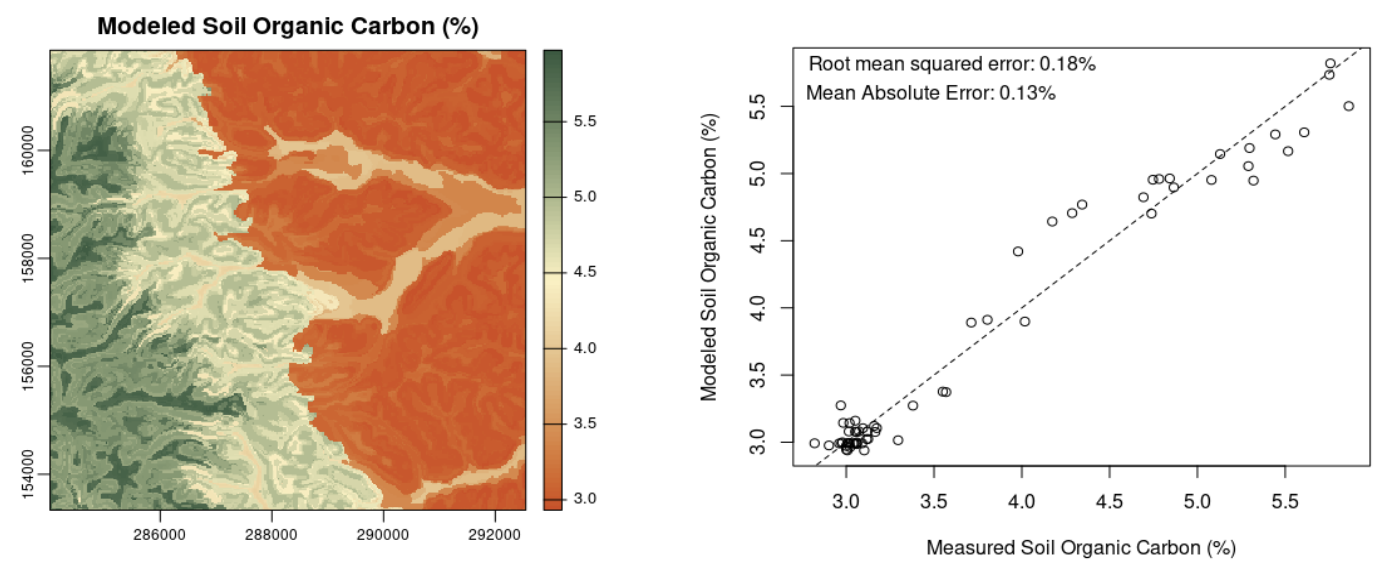

Figure 16: Modeled SOC and statistical evaluation using 62 independent observations.

\section{Miscellaneous}

The spatial signature only applies to classification units created from continuous variables. Thus, spatial signatures cannot be calculated for classification units that represent categorical variables, such as land use/land cover. In such cases, a one-hot encoding can be applied to produce binary layers for the units. These layers are considered the spatial signatures of the classification units. The code below shows the creation of binary layers for soil parent material units with dummies().
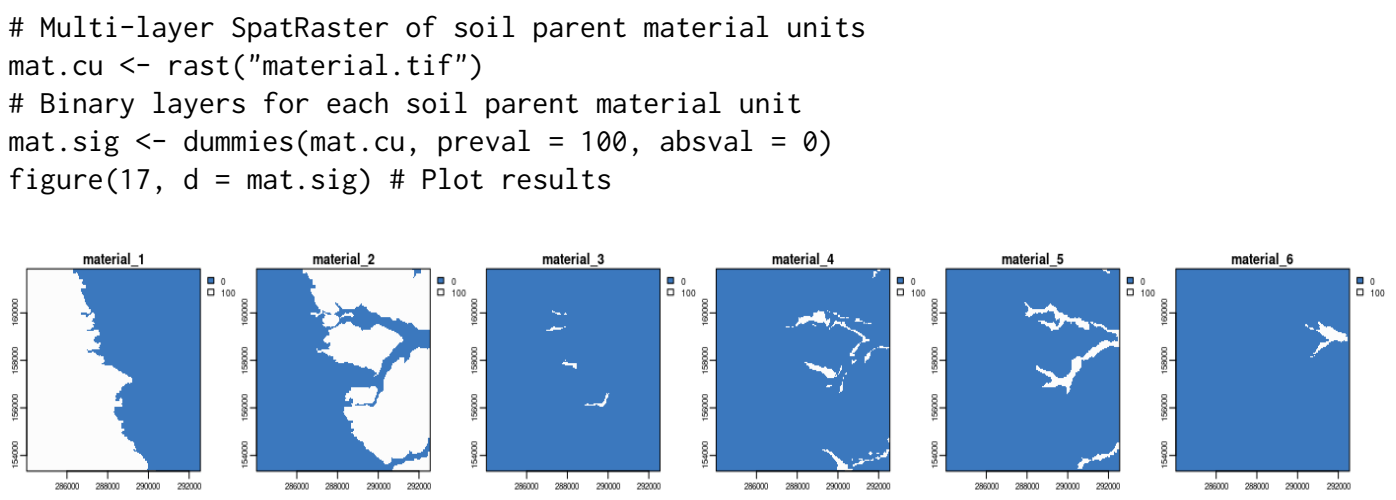

Figure 17: Binary layers as spatial signatures for soil parent material units.

The function $p l o t 3 D()$ produces interactive maps showing the 3-dimensional (XYZ) variability in raster layers representing continuous variables. The $X Y Z$ reference positions are obtained from 
a user-supplied elevation layer. For large raster layers (large spatial coverage and/or high spatial resolution), this function allows the option to decrease resolution and subset the data. The code below shows how plot3D() creates a 3D map for SOC, as modeled with engine().
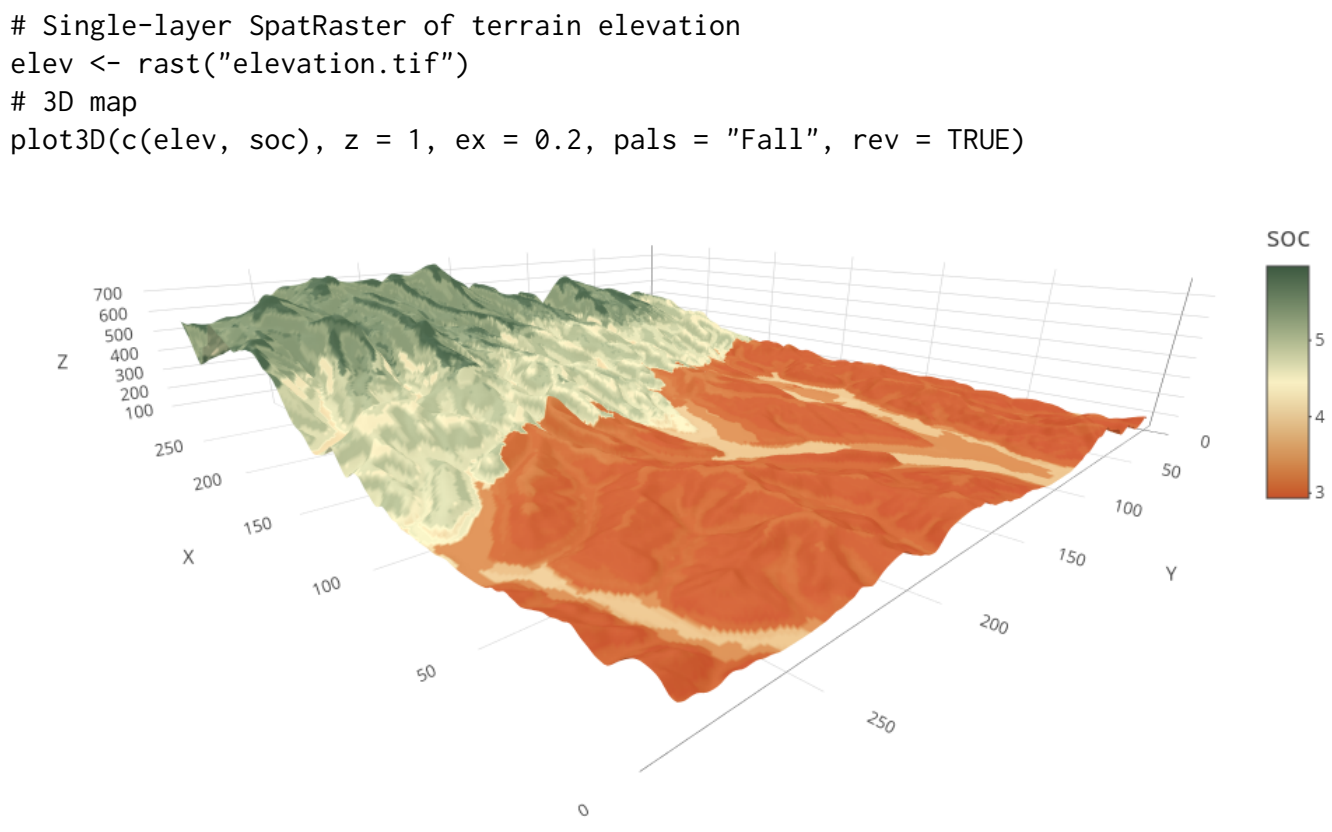

Figure 18: 3D map of modeled SOC (percent)

\section{Future versioning and Summary}

This work presented the rassta package for spatial stratification, sampling, and modeling of environmental phenomena within the $\mathrm{R}$ environment. Future versioning of the rassta package will focus on developing new approaches for spatial stratification. Stratification based on spatial intersection may not be feasible to implement in highly complex landscapes because these landscapes may require many (sets of) classification units to accurately represent the spatial variability of landscape factors, leading to over-stratification, and thus, greater demand for samples/observations to conduct predictive modeling based on landscape similarity. One plausible solution is the application of the stratification methods presented by Jasiewicz et al. (2015), Jasiewicz et al. (2018), Netzel and Stepinski (2015), Nowosad (2021), and Nowosad and Stepinski (2021). However, these methods have been purposely designed for studies with continental/global applications. Therefore, these methods should be adapted for rassta to tailor their application at local scales to allow for more precise representations of natural phenomena and their spatial variability. Another focus of versioning can be new functions to visualize the variability of response phenomena relative to the hierarchical structure represented by the stratification units. Lastly, future versioning of rassta should also consider the user's experiences to ensure its general applicability.

The core ideas implemented in the rassta package include the multi-scale, hierarchical landscape stratification based on spatial intersection, the application of non-parametric distribution estimators to define the typical landscape configuration of stratification units, and the use of spatially explicit landscape correspondence metrics for non-probability sampling and predictive modeling. Some of these ideas have previously been implemented in $\mathrm{R}$ through a few packages dedicated to the analysis of geospatial data. Nevertheless, rassta offers a unified, generalized framework to conduct multiple landscape stratification routines through a dedicated set of algorithms. Moreover, spatially-explicit information created with rassta, like stratification units, landscape similarity layers, and representative observations, can be embedded into statistically robust modeling approaches to optimize the analysis of environmental phenomena.

\section{Bibliography}

T. F. H. Allen and B. Starr. Hierarchy: Perspectives for Ecological Complexity. University of Chicago Press, 1982. URL https://doi.org/10.7208/chicago/9780226489711.001.0001. [p1,2] 
M. B. Araújo and A. Guisan. Five (or so) challenges for species distribution modelling. Journal of Biogeography, 33(10):1677-1688, 2006. URL https://doi .org/10.1111/j.1365-2699.2006.01584.x. [p1]

D. Arrouays, A. McBratney, J. Bouma, Z. Libohova, A. Richer-de Forges, C. Morgan, P. Roudier, L. Poggio, and V. Mulder. Impressions of digital soil maps: The good, the not so good, and making them ever better. Geoderma Regional, 20:e00255, 2020. URL https://doi . org/10.1016/j.geodrs. 2020.e00255. [p1]

M. P. Austin and P. C. Heyligers. New approach to vegetation survey design: gradsect sampling. Nature Conservation: Cost Effective Biological Surveys and Data Analysis, 5:31-36, 1989. [p3, 9, 10]

D. Baldwin, K. Naithani, and H. Lin. Combined soil-terrain stratification for characterizing catchmentscale soil moisture variation. Geoderma, 285:260-269, Jan. 2017. URL https://doi .org/10.1016/j . geoderma. 2016.09.031. [p1]

P. Burrough. Fuzzy mathematical methods for soil survey and land evaluation. Journal of Soil Science, 40(3):477-492, 1989. URL https://doi .org/10.1111/j.1365-2389.1989. tb01290.x. [p6]

G. Carpenter, A. N. Gillison, and J. Winter. DOMAIN: a flexible modelling procedure for mapping potential distributions of plants and animals. Biodiversity $\mathcal{E}$ Conservation, 2(6):667-680, 1993. URL https://doi.org/10.1007/bf00051966. [p6, 11]

W. Chang, J. Cheng, J. Allaire, C. Sievert, B. Schloerke, Y. Xie, J. Allen, J. McPherson, A. Dipert, and B. Borges. shiny: Web Application Framework for R, 2021. URL https: //CRAN. R-project.org/ package=shiny. $\mathrm{R}$ package version 1.6.0. [p7]

E. Chuvieco, I. Aguado, M. Yebra, H. Nieto, J. Salas, M. Pilar Martín, L. Vilar, J. Martínez, S. Martín, P. Ibarra, J. de la Riva, J. Baeza, F. Rodríguez, J. Molina, M. Herrera, and R. Zamora. Development of a framework for fire risk assessment using remote sensing and geographic information system technologies. Ecological Modelling, 221(1):46-58, Jan. 2010. URL https://doi .org/10.1016/ j.ecolmodel.2008.11.017. [p1]

J. Elith and J. R. Leathwick. Species distribution models: ecological explanation and prediction across space and time. Annual Review of Ecology, Evolution, and Systematics, 40:677-697, 2009. URL https://doi.org/10.1146/annurev. ecolsys.110308.120159. [p1]

J. Elith, M. Kearney, and S. Phillips. The art of modelling range-shifting species. Methods in Ecology and Evolution, 1(4):330-342, 2010. URL https://doi.org/10.1111/j.2041-210x.2010.00036.x. [p6]

W.-A. Flügel. Delineating hydrological response units by geographical information system analyses for regional hydrological modelling using PRMS/MMS in the drainage basin of the river Bröl, Germany. Hydrological Processes, 9(3-4):423-436, 1995. URL https://doi .org/10. 1002/hyp. 3360090313. [p2]

A. Gillison. Gradient oriented sampling for resource surveys - the gradsect method. Survey Methods for Nature Conservation, 2:349-374, 1983. [p10]

A. Gillison and K. Brewer. The use of gradient directed transects or gradsects in natural resource surveys. Journal of Environmental Management, 20:103-127, Jan. 1985. [p10]

K. Glinka. Dokuchaev's ideas in the development of pedology and cognate sciences. Russian Pedological Investigations, 1, 1927. [p2]

A. Guisan and N. Zimmermann. Predictive habitat distribution models in ecology. Ecological Modelling, 135(2):147-186, 2000. URL https://doi .org/10.1016/S0304-3800(00)00354-9. [p1,10]

J. Ham, Y. Chen, M. Crawford, and J. Ghosh. Investigation of the random forest framework for classification of hyperspectral data. IEEE Transactions on Geoscience and Remote Sensing, 43(3): 492-501, 2005. URL https://doi.org/10.1109/tgrs.2004.842481. [p1]

G. Heuvelink and R. Webster. Modelling soil variation: past, present, and future. Geoderma, 100(3): 269-301, 2001. URL https://doi.org/10.1016/S0016-7061(01)00025-8. [p1]

R. J. Hijmans. terra: Spatial Data Analysis, 2021. URL https: //CRAN. R-project. org/package=terra. $\mathrm{R}$ package version 1.3-4. [p1]

R. J. Hijmans, S. Phillips, J. Leathwick, and J. Elith. dismo: Species Distribution Modeling, 2020. URL https://CRAN.R-project.org/package=dismo. R package version 1.3-3. [p6] 
A. Hirzel, V. Helfer, and F. Metral. Assessing habitat-suitability models with a virtual species. Ecological Modelling, 145(2-3):111-121, 2001. URL https://doi.org/10.1016/s0304-3800(01)00396-9. [p6]

B. Hudson. The soil survey as paradigm-based science. Soil Science Society of America Journal, 56(3): 836-841, 1992. URL https://doi.org/10.2136/sssaj1992.03615995005600030027x. [p2]

J. Jasiewicz, P. Netzel, and T. Stepinski. GeoPAT: A toolbox for pattern-based information retrieval from large geospatial databases. Computers E Geosciences, 80:62-73, July 2015. URL https: //doi . org/10.1016/j. cageo.2015.04.002. [p1, 13]

J. Jasiewicz, T. Stepinski, and J. Niesterowicz. Multi-scale segmentation algorithm for pattern-based partitioning of large categorical rasters. Computers $\mathcal{E}$ Geosciences, 118:122-130, Sept. 2018. URL https://doi.org/10.1016/j.cageo.2018.06.003. [p13]

L. Kaufman and P. Rousseeuw. Finding Groups in Data: An Introduction to Cluster Analysis. John Wiley \& Sons, 1990. [p4]

S. Knick and D. Dyer. Distribution of black-tailed jackrabbit habitat determined by GIS in Southwestern Idaho. The Journal of Wildlife Management, pages 75-85, 1997. URL https://doi.org/10.2307/ 3802416. [p11]

T. Kohonen. The self-organizing map. Proceedings of the IEEE, 78(9):1464-1480, 1990. URL https: //doi.org/10.1016/s0925-2312(98)00030-7. [p4]

B. Leroy, C. Meynard, C. Bellard, and F. Courchamp. virtualspecies, an R package to generate virtual species distributions. Ecography, 39(6):599-607, 2016. URL https://doi .org/10.1111/ecog. 01388. [p6]

R. MacMillan, W. Pettapiece, S. Nolan, and T. Goddard. A generic procedure for automatically segmenting landforms into landform elements using DEMs, heuristic rules and fuzzy logic. Fuzzy Sets and Systems, 113(1):81-109, 2000. URL https://doi.org/10.1016/S0165-0114(99)00014-7. [p6]

A. McBratney, M. L. Mendonça Santos, and B. Minasny. On digital soil mapping. Geoderma, 117(1-2): 3-52, 2003. URL https://doi.org/10.1016/S0016-7061(03)00223-4. [p1]

K. McSweeney, B. K. Slater, R. David Hammer, J. C. Bell, P. E. Gessler, and G. W. Petersen. Towards a new framework for modeling the soil-landscape continuum. Factors of Soil Formation: A Fiftieth Anniversary Retrospective, 33:127-145, 1994. URL https://doi .org/10.2136/sssaspecpub33.c8. [p2, 3]

P. Netzel and T. Stepinski. Pattern-based assessment of land cover change on continental scale with application to NLCD 2001-2006. IEEE Transactions on Geoscience and Remote Sensing, 53(4):1773-1781, 2015. URL https://doi.org/10.1109/TGRS.2014.2348715. [p1,13]

J. Nowosad. Motif: an open-source R tool for pattern-based spatial analysis. Landscape Ecology, 36(1): 29-43, 2021. URL https://doi.org/10.1007/s10980-020-01135-0. [p1, 13]

J. Nowosad and T. Stepinski. Pattern-based identification and mapping of landscape types using multi-thematic data. International Journal of Geographical Information Science, 35(8):1634-1649, Mar. 2021. URL https://doi.org/10.1080/13658816.2021.1893324. [p13]

S. J. Park and N. Van De Giesen. Soil-landscape delineation to define spatial sampling domains for hillslope hydrology. Journal of Hydrology, 295(1-4):28-46, 2004. URL https://doi .org/10. 1016/j . jhydrol.2004.02.022. [p1]

E. Pebesma. Simple features for R: Standardized support for spatial vector data. The R Journal, 10(1): 439-446, 2018. URL https://doi .org/10.32614/RJ-2018-009. [p1]

R. Pike. The geometric signature: quantifying landslide-terrain types from digital elevation models. Mathematical Geology, 20(5):491-511, 1988. URL https: //doi .org/10.1007/BF00890333. [p6]

R. Pike and W. Rozema. Spectral analysis of landforms. Annals of the Association of American Geographers, 65(4):499-516, 1975. URL https://doi.org/10.1111/j.1467-8306.1975. tb01058.x. [p6]

M. Rodrigues, S. Costafreda-Aumedes, C. Comas, and C. Vega-García. Spatial stratification of wildfire drivers towards enhanced definition of large-fire regime zoning and fire seasons. Science of the Total Environment, 689:634-644, Nov. 2019. URL https://doi .org/10.1016/j. scitotenv. 2019.06. 467. [p1] 
J. Schmidt, P. Tonkin, and A. Hewitt. Quantitative soil - landscape models for the Haldon and Hurunui soil sets, New Zealand. Soil Research, 43(2):127, 2005. URL https://doi . org/10.1071/sr04074. [p11]

P. Scull, J. Franklin, O. Chadwick, and D. McArthur. Predictive soil mapping: a review. Progress in Physical Geography, 27(2):171-197, 2003. URL https://doi .org/10.1191/0309133303pp366ra. [p1]

D. Sui. A fuzzy GIS modeling approach for urban land evaluation. Computers, Environment and Urban Systems, 16(2):101-115, 1992. URL https://doi .org/10. 1016/0198-9715(92)90022- J. [p6]

R. Tibshirani, G. Walther, and T. Hastie. Estimating the number of clusters in a data set via the gap statistic. Journal of the Royal Statistical Society: Series B (statistical Methodology), 63(2):411-423, 2001. URL https://doi.org/10.1111/1467-9868.00293. [p4]

K. Watrous, T. Donovan, R. Mickey, S. Darling, A. Hicks, and S. Von Oettingen. Predicting minimum habitat characteristics for the Indiana bat in the Champlain Valley. The Journal of Wildlife Management, 70(5):1228-1237, 2006. URL https://doi .org/10.2193/0022-541X(2006) 70[1228: PMHCFT]2.0. C0; 2. $[\mathrm{p} 11]$

R. Wehrens and J. Kruisselbrink. Flexible self-organizing maps in kohonen 3.0. Journal of Statistical Software, 87(1):1-18, 2018. URL https://doi.org/10.18637/jss.v087.i07. [p4]

K. J. Wessels, S. Van Jaarsveld, J. D. Grimbeek, and M. J. Van der Linde. An evaluation of the gradsect biological survey method. Biodiversity $\mathcal{E}$ Conservation, 7(8):1093-1121, 1998. URL https: //doi.org/10.1023/a:1008899802456. [p10]

A. West, S. Kumar, C. Brown, T. Stohlgren, and J. Bromberg. Field validation of an invasive species maxent model. Ecological Informatics, 36:126-134, Nov. 2016. URL https://doi.org/10.1016/j. ecoinf.2016.11.001. [p1,10]

L. A. Zadeh. Fuzzy sets. Information and Control, 8(1):338-353, 1965. URL https://doi .org/10.1142/ 9789814261302_0001. [p6]

A. X. Zhu. A similarity model for representing soil spatial information. Geoderma, 77(2-4):217-242, 1997. URL https://doi .org/10.1016/s0016-7061(97)00023-2. [p1,6,11]

A. X. Zhu and L. Band. A knowledge-based approach to data integration for soil mapping. Canadian Journal of Remote Sensing, 20(4):408-418, Dec. 1994. URL https: // doi .org/10. 1080/07038992.1994. 10874583. [p11]

A. X. Zhu, L. Yang, B. Li, C. Qin, E. English, J. Burt, and C. Zhou. Purposive sampling for digital soil mapping for areas with limited data. In Digital Soil Mapping with Limited Data, pages 233-245. Springer, 2008. URL https://doi.org/10.1007/978-1-4020-8592-5_20. [p10]

A. X. Zhu, F. Qi, A. Moore, and J. Burt. Prediction of soil properties using fuzzy membership values. Geoderma, 158(3-4):199-206, Sept. 2010. URL https://doi .org/10.1016/j . geoderma.2010.05.001. [p11]

Bryan A. Fuentes

University of Arkansas

Department of Crop, Soil, and Environmental Sciences

Fayetteville, Arkansas

ORCiD: 0000-0003-3506-7101

bafuente@uark. edu

Minerva J. Dorantes

University of Arkansas

Department of Crop, Soil, and Environmental Sciences

Fayetteville, Arkansas

ORCiD: 0000-0002-2877-832X

mjdorant@uark. edu

John R. Tipton

University of Arkansas

Department of Mathematical Sciences

Fayetteville, Arkansas

ORCiD: 0000-0002-6135-8141

jrtipton@uark.edu 